\title{
Modeling Knot Geometry in Norway Spruce from Industrial CT Images
}

\author{
Jean-Philippe Andreu and Alfred Rinnhofer \\ JOANNEUM RESEARCH, Institute of Digital Image Processing \\ Wastiangasse 6, A-8010 Graz, AUSTRIA \\ \{jean-philippe.andreu, alfred.rinnhofer\}@joanneum.at
}

\begin{abstract}
For spruce (Picea abies (L.) Karst.), as with most other species, the value of a log without major defects like decay or compression wood is to great extent determined by its knot structure. That is the reason why sawyers, aiming at the optimal utilization of a $\log$, were naturally interested in the knots, their number, position, diameter and length. X-ray computer tomography (CT) scanning is able to detect internal density variations like knots. To use this information for a computer based optimization process, it is necessary to model different defects by geometric descriptions which are easy to handle. The purpose of this study is to show how to model knots in spruce logs from nondestructive measurements using industrial CT and automatic image analysis processes. A comparison between the results of these methods and the results of a destructive method gives an experimental evaluation of our approach.
\end{abstract}

\section{Introduction}

Internal structures such as knots, cracks, decay and other defects ultimately determine the value of a log for the lumber industry. It has been shown that acquiring a detailed knowledge of the location and size of internal defects prior to the first cut into the log is estimated to lead to potential gains in lumber value $[3,5,7]$.

\subsection{Industrial CT Scanning}

For about 20 years, research has been conducted to find non-destructive technologies to scan logs for internal defects. X-ray technology was identified as one of the most promising technology to image the internal structure of logs compared to other techniques using Gamma rays, nuclear magnetic resonance, microwaves, ultrasounds, vibrations and longitudinal stress waves. Good overviews and descriptions of these different technologies can be found in $[6,11]$. Because of similar density ranges between human tissues and wood, X-ray technologies (including CT) developed for medical purposes were investigated for wood scanning.

Even though medical CT scanners have proven their ability to produce images of really high quality, trials using medical CT scanners for scanning logs have been limited to small logs. Medical CT scanners have, by design, strict limits (in terms of 
duty cycle, speed and gantry size) which are hindering these machines to scan normal sized logs all day long within the harsh industrial environment of a sawmill. CT scanners developed for airport security purposes (metal and explosive detection) have bridged the gap between the medical and the industrial CT application domains. These scanners [10] work like their medical cousins but their x-ray systems are designed for operating at a duty cycle of $100 \% 24$ hours a day. They allow a bigger gantry than medical CT scanners and can be operated at a higher speed. But such changes in design result in a lower image quality compared to the high resolution images a medical CT scanner can provide.

\subsection{Related Work on Knot Modeling}

Modeling a 3D object out of real-world measurements aims at encapsulating the shape of a 3D object into a parametrical mathematical formulation. Parametric descriptions dramatically reduce the amount of data (i.e. measurements) to handle. They translate the 3D objects into a continuous function while keeping the representation detailed.

While most of the researchers put effort on precisely classifying pixels either as defect (belonging to a specific defect type such as knot, void, rot, etc...) or non-defect, less effort was put in designing models for wood defects. J.E. Aune [2] and M. Samson [9] developed geometrical models to describe knots within logs. Grundberg [6] developed a model for Scots pine knots. This model describes a knot as three functions of the radial distance to the pith (knot diameter, tangential and longitudinal position). This model can not describe the longitudinal position of knots changing direction from upwards to downwards since the function used for modeling the longitudinal position is an increasing function. Oja [8] later improved this model for Norway spruce by using a complicated non-linear function (a mixture of $\ln (x)$ and $\tan (x)$ ) for describing the longitudinal position of the knots and finally concluded that there is no significant difference in accuracy between his model and the one developed by Grundberg [6].

\section{Preprocessing and Segmentation of the CT images}

Spruce logs were scanned using an Invision CTX 2500 CT scanner with a pixel resolution of $1.55 \mathrm{~mm}$ by $1.55 \mathrm{~mm}$, an intensity resolution of 12 bits per pixel (i.e. 4096 gray levels) and an image size of 512x512 pixels. An image was taken every 20 $\mathrm{mm}$ with a feeding speed of $1.5 \mathrm{~m} / \mathrm{min}$. Practically, this is too slow for volume oriented high-speed sawmills, but sufficient for quality oriented sawmills.

As described in [1], we first localize the pith by considering it as the center of the annual rings (segmented by improving the clarity of their ridge and valley structures with the help of local orientation and frequency information).

The density distribution of log structures tend to be very similar across logs of the same specie. In a typical CT image of spruce, the pixels can be classified into four classes based on gray-level value. From the lowest to the highest gray level value they correspond to pixels from: (1) cracks, (2) heartwood, (3) sapwood and (4) knots. Still, the gray levels of one class can overlap with those of adjacent classes. Especially, 
sapwood and knots are difficult to distinguish from each other. In the absence of internal defects, the corresponding CT image slice exhibits gray-level variations resulting solely from the ring structure. Therefore in a second step we remove the fine structure of the annual rings by smoothing them out using simple Gaussian filtering. To classify the pixels into the aforementioned classes we then use a multi-modal histogram thresholding method [4].

\section{Knot modeling}

Corresponding knot regions in successive CT images give support for modeling a knot in 3D. Regions in successive CT images are grouped together depending on their distance to the pith and the direction of their principal axis in the CT image plane. Each group representing a series of $2 \mathrm{D}$ regions that are spatially connected across CT images is then modeled as a single knot in 3D. Note that some knots are lacking of 3D support (e.g. knots only appearing on a single CT image). Each knot is mathematically modeled by a curve in 3D (spine) along which a $2 \mathrm{D}$ cross-section (a planar patch) is swept. Ideally the sweeping of a 2D shape along a path in 3D creates a volume (swept volume or generalized cylinder). The shape of the $2 \mathrm{D}$ cross-section is fixed but can be differently scaled at each point of the spine. If the function representing the spine lays on a plane, each knot is having a symmetry plane and the spine function can be describe as a $2 \mathrm{D}$ function within this symmetry plane. The origin of the spine function is located at the pith of the CT image where the knot first appeared. The knot symmetry plane is perpendicular to the CT image plane, passes through the pith and has an angle $\alpha$ with the CT image plane. The following figure (Fig.1) shows such a knot model (textured and wire-framed rendered):

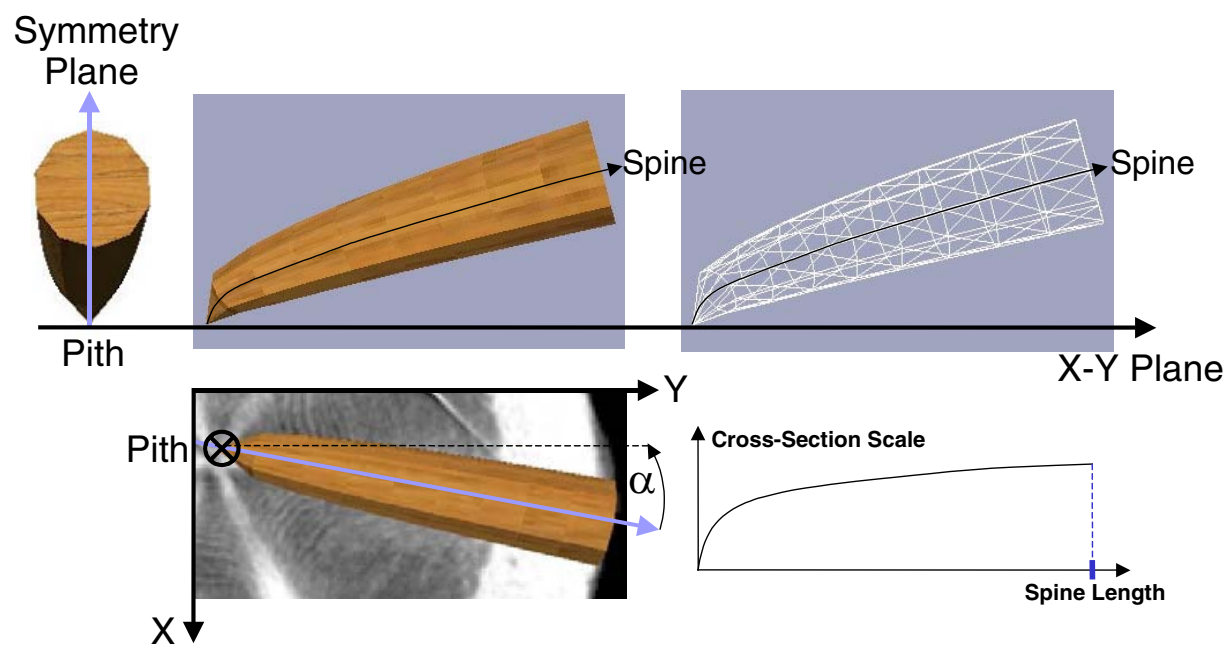

Fig. 1. Knot model defined by: a symmetry plane (in gray), a planar spine function, its origin (Pith) in the CT image plane (X-Y), the angular $(\alpha)$ position of the symmetry plane with respect to the $\mathrm{X}-\mathrm{Y}$ plane, a scaling function of a $2 \mathrm{D}$ cross-section. 
The planar (in the knot symmetry plane) spine function is described by:

Spine $(r)=a \sqrt{r}+b r, r \in[0$, KnotLength $] \rightarrow[0$, KnotHeight $]$

and the scaling function of a 2D cross-section (a circle in our case) by:

Scale $(r)=c \ln (r+1), r \in[0$, KnotLength $] \rightarrow[0$, KnotMaxDia meter $]$

In the equations (1) and (2), the variable $r$ defines the radial distance from the pith.

Note that our knot model is similar to VRML97 extrusions [12] which are also based on a two dimensional cross-section extruded along a three dimensional spine. The only difference between our knot model and a VRML extrusion lays in the piecewise linearity of the 2D cross-section and the 3D spine of the VRML shape. VRML extrusions are convenient for 3D graphics rendering and their intersection with a cutting plane (intersection of a knot with a sawing plane) can be easily computed by computational geometry algorithms.

\section{Experimental Results}

The aforementioned procedures for detecting and modeling the knots were experimentally validated on four logs, two of them having knots changing direction from upwards to downwards. The logs were scanned and cut into $23 \mathrm{~mm}$ parallel boards. The boards were imaged with an optical line scan camera with a pixel resolution of $1 \mathrm{~mm}$ by $1 \mathrm{~mm}$. The position and the maximum diameter of the knots appearing on the board faces were manually recorded and treated as ground truth. After automatically detecting and modeling the 3D knots from the CT data, their intersection with the cutting plane corresponding to each board face were computed. For visual control and validation the intersections between the 3D knots and the cutting planes were then overlaid onto the scanned images of the board faces (Fig. 2).

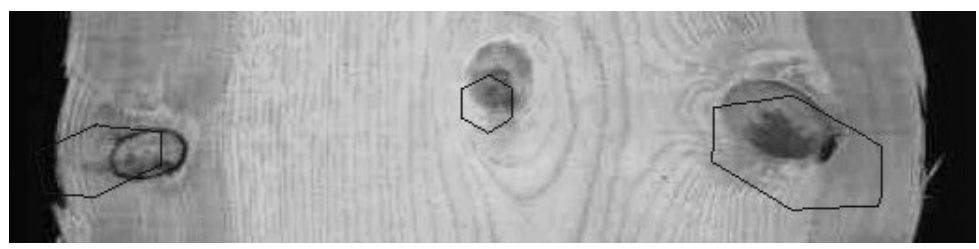

Fig. 2. Part of an optically scanned board with knots and detected knots overlaid

The detection rate and false alarm rate were computed. The detection rate is defined as the ratio of the number of true knots detected to the total number of true knots appearing on the board faces. The false alarm rate is defined as the ratio of the number of false knots detected to the total number of knots detected (true and false). The detection and false alarm rates for knots having a diameter of at least $10 \mathrm{~mm}$ averaged to $96 \%$ and $10 \%$ respectively. If all knots were considered, the detection rate falls to $73 \%$ and the false alarm rate reaches $13 \%$. The following table (Table 1) shows the accuracy and position of the knot modeling with respect to the knot size. 
Table 1. Knot model accuracy for knots whose diameter $>10 \mathrm{~mm}$

\begin{tabular}{lcc}
\hline Knot Parameter & Mean Value & Standard Deviation \\
\hline Angular position: $\alpha$ & $1.9^{\circ}$ & $2.9^{\circ}$ \\
Elevation position & $0.9 \mathrm{~mm}$ & $10.4 \mathrm{~mm}$ \\
Diameter & $0.7 \mathrm{~mm}$ & $10.1 \mathrm{~mm}$ \\
\hline
\end{tabular}

\subsection{Thin knots}

The spatial resolution of the scanner in the plane perpendicular to the feeding direction is fixed by mechanical constraints (number of sensors on the detector array, sensor size, focus size on the anode, gantry diameter, etc...). The transversal feeding speed of the scanner fix the volume to integrate: the quicker the speed, the more volume, the lower the contrast (averaging effect). Limitations also come from the data acquisition. The whole spiral is not completely taken into account for reconstruction leaving in "gaps" into the data. These limitations result in a poor image quality, making thin knots difficult to detect. Thin structures are also easily destroyed by image processing (filtering, morphology, etc...). Whenever possible we apply algorithms tuning themselves to the quality of the image and preserving thin structures. This ability to adapt to the image quality is a great advantage. If the quality of the images increases, thin knots will be easier to detect.

\subsection{Knots ending in the Sapwood}

Due to its high humidity the sapwood is characterized in CT images by high densities which tend to blur neighboring lower density structures. Due to the low spatial resolution of our industrial CT scanner, it is very difficult to detect structures within the sapwood. We assume that knots reaching the sapwood will reach the surface of the trunk. Unfortunately, this assumption does not always hold.

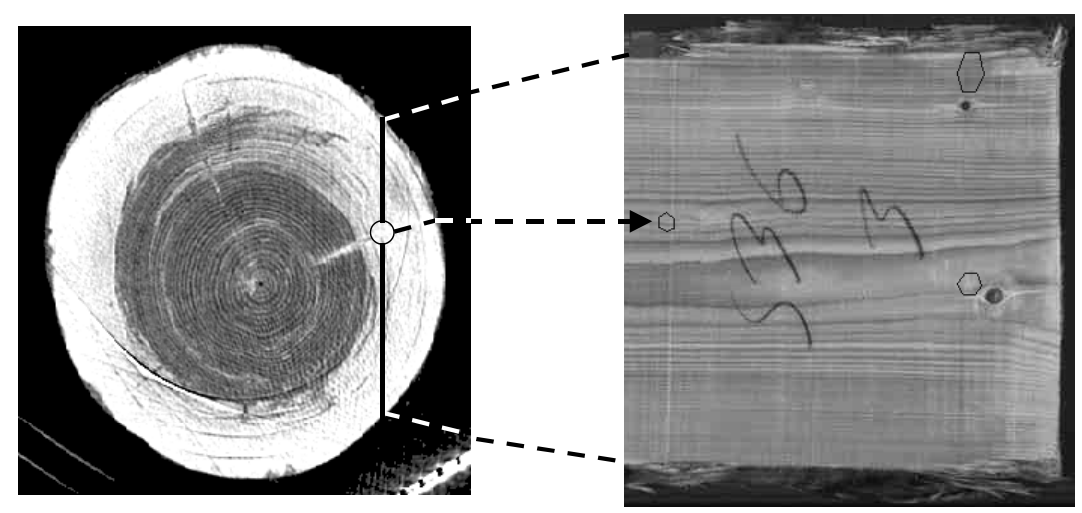

Fig. 3. Knot ending in the sapwood. Left: knot reaching the sapwood. Right: the board face corresponding to the cut along the vertical line does not contain a knot at the predicted position. 
Figure 3 depicts the situation where we detect a knot reaching the sapwood and therefore assuming it will reach the surface. In reality it is ending within the sapwood.

\section{Conclusion}

We have developed a model for knots in Norway spruce logs using industrial CT scanning and automatic image analysis algorithms. Experimental results show a good detection rate and accuracy of the model for knots having a diameter of at least $10 \mathrm{~mm}$. The present industrial scanner system we used has limitations in term of resolution and speed. We hope that new generations of industrial CT scanners will overcome these limitations and enable fully automated systems for optimized log breakdown.

\section{References}

1. Andreu, J.Ph. and Rinnhofer, A.: "Enhancement of annual rings on industrial CT images of logs", Proc. ICPR2002, Vol. III (2002), 261-264.

2. Aune, J.: "An X-ray Log-Scanner for Sawmills", Proc. of the 2nd Int. Workshop on Scanning Technology and Image Processing on Wood (1995), 51-65.

3. Brdicko, J., Orbay, L. and Tong, Q.: "Using External and Internal Log Characteristics for Log Breakdown Optimization", Proc. of the 5th Int. Conference on Image Processing and Scanning of Wood (2003), 115-124.

4. Chang, J.-H., Fan K.-C. and Chang Y.-L.: "Multi-modal gray-level Histogram Modeling and Decomposition", Image and Vision Computing, 20 (2002) 203-216.

5. Chang, S. J.: "Hardwood Sawing Optimization based on CT Scanning of Internal Defects", Proc. of the 5th Int. Conference on Image Processing and Scanning of Wood (2003), 125130.

6. Grundberg, S.: "Scanning for internal defects in logs", Ph.D. thesis, Lulea Univ. of Tech., (1994) ISSN 0280-8242.

7. Hodges, D.G., Anderson, W.C. and Mac Millin, C.W. : "The economic potential of CT scanners for hardwood sawmills", Forest Products Journal 40(3), (1990) 65-69.

8. Oja, J.: "X-ray Measurement of Properties of Saw Logs", Ph.D. thesis, Lulea Univ. of Tech., (1999) ISSN 1402-1544.

9. Samson, M.: "Modeling of knots in logs", Wood Science and Tech., 27 (1993), 429-437.

10. Schmoldt D.L., Scheinman E., Rinnhofer A. and Occeña L.G., "Internal log scanning: Research to reality", Proc. of the Hardwood Research Council Meeting, (2000).

11. Skatter, S.: "Non destructive determination of the external shape and the internal structure of logs-Possible technologies for use in the sawmills", Ph.D. thesis, Agricultural Univ. of Norway, ISBN 82-575-0352-5 (1998).

12. VRML97, The Virtual Reality Modeling Language, http://www.web3d.org/Specifications/ 